\title{
Reliability Analysis on the Rectangular Orthotropic Membrane Under Concentrated Impact Load
}

\author{
Zhoulian ZHENG*, Xiaomeng XIE, Dong LI, Hao WANG \\ College of Civil Engineering \\ Chongqing University \\ Chongqing, China \\ e-mail: zhengzl@cqu.edu.cn
}

\begin{abstract}
This paper studies the computational method of the reliability of rectangular orthotropic membrane with four edges fixed under stochastic concentrated impact load. Based on the explicit equations of deflection response obtained in the previous work of our team, the reliability index and failure probability of the architectural membrane under concentrated impact load are obtained via the advanced first-order secondmoment method(AFOSM). In addition, an experiment on stochastic impact load was carried out. Based on the principle of least-squares method, the impact speed is fitted to a Gaussian distribution with specific mathematical characteristics. The data are processed by Monte-Carlo method and the results are compared with the theoretical results calculated by AFOSM. The variation rules and differences of the results obtained by two methods are analyzed. This study provides a theoretical reference for the vibration control of membrane structures and the research on the failure of membrane due to hail and other disasters.
\end{abstract}

Keywords-membrane structure; reliability; dynamic; impact; orthotropic

\section{INTRODUCTION}

Building membranes act both as structure and as cladding, reducing the weight, cost, and environmental impact of the construction. Membrane structures have fashionable architectural performances as well as a high level of structural stability and durability. However, due to its light weight and low rigidity, the architectural membrane has the characteristics of large deformation, geometric nonlinearity, small damping and low natural frequency, making it particularly sensitive to the external load. In addition, due to the randomness of the load caused by the natural climate, stress concentration may occur once the membrane fails or tears partly, which may endanger the safety of the entire structure. Therefore, studying the reliability of membrane structure under the stochastic impact load is of great significance to the structural design, construction and maintenance. Therefore, to prevent such kinds of engineering accidents from happening and reduce the loss membrane structure suffers, the related researches are very necessary.

In 2004, Farid Khan [1] studied the nonlinear behaviour of membrane type electromagnetic energy harvester under harmonic and random vibrations. In 2007, Jie Li [2] researched the equivalent extreme-value event and evaluation of the structural system reliability, and proved his approach is of satisfactory accuracy and applicable to the structural reliability analysis of various structures. In 2008, J.W. Fox [3][4] studied the dynamic characteristics and dynamic electron load of dielectric elastic membranes. In 2010, Liu Chang-jiang [5] deduced the governing equations of geometric nonlinear free and forced vibration of orthotropic rectangular membrane structures. The LPD perturbation method and KBM perturbation method were used to obtain the analytical solutions of the deflections of the membranes without damping and damping. In 2012, P.D. Gosling [6] studied a reliability approach for membrane structure with numerical simulation. In 2015, Weiju Song [7] obtained the functional relation between different structure parameters and the natural frequency of the thin plates, provide some computational basis for the vibration control and dynamic design of stochastic parameter structures.

In this paper, methods of calculating the reliability of an orthotropic rectangular membrane under stochastic concentrated impact load are studied and the applicability of first-order second-moment method in the calculation of the reliability of the membrane structure is proved. According to the experiment, a stochastic load model is established via least-squares method. Monte-Carlo method is used to process the data to get the reliability index and failure probability of the membrane structure. The change regulations of the reliability under different parameters are analyzed with these two methods. Conclusions have some practical significance on the vibration control design of the membrane structure, which helps to prevent the membrane structure from tearing accidently due to the excessive response of the vibration deflection resulting from the stochastic dynamic load.

\section{CALCULATION METHOD OF RELIABILITY INDEX AND FAILURE PROBABILITY}

According to the previous work of our research team, the response function of rectangular orthotropic membrane under concentrated impact load was obtained via the stochastic vibration theory and the perturbation method[8]: 


$$
\begin{aligned}
& w(x, y, t)=\sum_{m=1}^{\infty} \sum_{n=1}^{\infty} T_{m n}(t) \cdot W_{m n}(x, y)=\sum_{m=1}^{\infty} \sum_{n=1}^{\infty}\left(\frac{4 M \delta v \sin \left(\frac{m \pi x_{0}}{a}\right) \sin \left(\frac{n \pi y_{0}}{b}\right) e^{-\beta t}}{\rho a b \sqrt{\omega_{0}^{2}-\beta^{2}}} \cdot \sin \left(\sqrt{\omega_{0}^{2}-\beta^{2}}\right) t\right.
\end{aligned}
$$

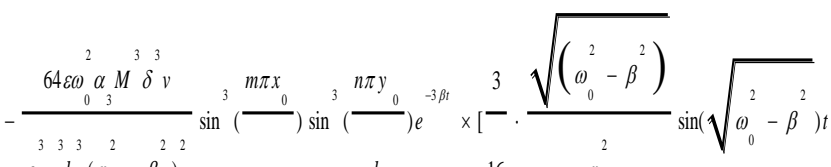

$$
\begin{aligned}
& \begin{array}{ccccc}
\rho a b(\omega-\beta) & a & b & 16 & \omega_{0}
\end{array} \\
& +\left(\frac{3}{16 \beta}-\frac{3 \beta}{16 \omega_{0}^{2}}\right) \cos \left(\sqrt{\omega_{0}^{2}-\beta^{2}}\right) t+\left(\frac{\sqrt{\omega_{0}^{2}-\beta^{2}}}{16 \omega_{0}^{2}}-\frac{\sqrt{\omega_{0}^{2}-\beta^{2}}}{2 \cdot\left(-12 \beta^{2}+16 \omega_{0}^{2}\right)}\right) \sin \left(3 \sqrt{\omega_{0}^{2}-\beta^{2}}\right) t
\end{aligned}
$$

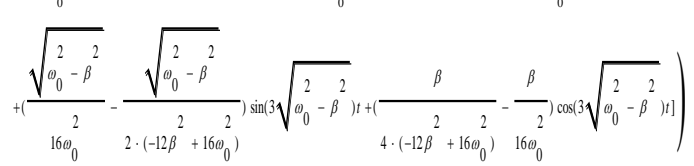

$$
\begin{aligned}
& \cdot \sin \left(\frac{m \pi x}{a}\right) \sin \left(\frac{n \pi y}{b}\right) \\
& \omega_{0}^{2}=\frac{m^{2} \pi^{2} b^{2} N_{0 x}+n^{2} \pi^{2} a^{2} N_{0 y}}{\rho a^{2} b^{2}} \alpha_{3}=\frac{6 a b m^{2} n^{2} \pi^{2}\left(\alpha_{1}+\beta_{1}\right)}{h\left(m^{2} b^{2} N_{0 x}+n^{2} a^{2} N_{0 y}\right)} \quad \varepsilon=\frac{h^{2}}{a b} \quad \beta=\frac{c}{2 \rho} \\
& \alpha_{1}=\frac{E_{2} n^{2} a^{2}}{32 m^{2} b^{2}} \beta_{1}=\frac{E_{1} m^{2} b^{2}}{32 n^{2} a^{2}}
\end{aligned}
$$

And $N_{0 x}$ is the pre-tension in the $x$ direction; $N_{0 y}$ is the pre-tension in the y direction; $\rho$ is the density of the membrane; $h$ is the thickness of the membrane; $\mathrm{E}_{1}$ is the elastic modulus in the warp direction; $\mathrm{E}_{2}$ is the elastic modulus in the weft direction; $M$ is the mass of impact body; $\delta v$ is the impact velocity。

\section{A. Advanced first-order second-moment method}

The membrane structure is easily deformed under impact load, resulting in laceration in the membrane surface. Due to the excessive deflection, a large amount of ponding and dirt are formed on the surface of the membrane, which is unfavorable to the normal function of the membrane structure. Different regions have different regulations for the maximum deflection limit of deflection in the design of membrane structure. Among them, the regulation in the Shanghai area of China [9] stipulate that the maximum deflection under the first type of load effect combination should not be larger than $1 / 250$ of the span or $1 / 125$ of the cantilever length. Therefore, the design of the membrane structure treats deflection as a key parameter, the use of the maximum deflection value and limit the establishment of limit state equation:

$$
Z=w_{B}-w_{t o p}=g\left(x_{1}, x_{2}, \cdots, x_{n}\right)
$$

Where $w_{t o p}$ is the maximum deflection value, $w_{B}$ is the deflection threshold, $x_{i}$ is the $n$th Basic design variables, $g$ is the $n$th performance function. The structure fails when $Z<0$.

Based on the explicit performance function, the advanced first-order second-moment method (AFOSM) is used to solve the reliability problem. The Taylor series is used to expand the function at the most probable failure point and keep the first two terms. The failure probability of the linear function is used to approximate the failure probability of the original nonlinear function and the reliability index is calculated.

The design point needs to be calculated by iterative calculation, and the mean value of the variable can be used as the initial design point $x^{*}$ :

$$
x^{*}=\mu_{x}
$$

The sensitivity coefficient of $x_{i}$ is as follow:

$$
\alpha_{x_{i}}=\cos \theta_{x_{i}}=-\frac{\frac{\partial g\left(x^{*}\right)}{\partial x_{i}} \sigma_{x_{i}}}{\sqrt{\sum_{i=1}^{n}\left[\frac{\partial g\left(x^{*}\right)}{\partial x_{i}}\right]^{2} \sigma_{x_{i}}^{2}}}
$$

Reliability index of the structure:

$$
\beta=\frac{\mu_{Z}}{\sigma_{Z}}=\frac{g\left(x^{*}\right)+\sum_{i=1}^{n} \frac{\partial g\left(x^{*}\right)}{\partial x_{i}}\left(\mu_{x_{i}}-x_{i}^{*}\right)}{\sqrt{\sum_{i=1}^{n}\left[\frac{\partial g\left(x^{*}\right)}{\partial x_{i}}\right]^{2} \sigma_{x_{i}}^{2}}}
$$

The new design point can be calculated iteratively:

$$
x_{i}^{*}=\mu_{x_{i}}+\beta \sigma_{x_{i}} \cos \theta_{x_{i}}
$$

Substituting the new design points into Eq.4-Eq.6, until a stable reliability index and design point are obtained.

Thus, the probability of failure is:

$$
P_{f}=P(Z<0)=\Phi(-\beta)
$$

Where ${ }^{\Phi(\cdot)}$ is the cumulative distribution function of a standard normal variable.

\section{B. Monte-Carlo method}

According to the Law of Large Numbers, Monte-Carlo method infer the parent's statistical laws by the probability 
characteristic of sample. When sample data is of large quantity, the calculation result is relatively accurate. For the fact that there is little research on the reliability of the membrane structure currently, Monte-Carlo method can be used to calculate its reliability and to verify the effectiveness of other methods.

The probability of safety is calculated as follows:

$$
P_{s}=1-P_{f}
$$

The calculation of the limit state equation is based on basic design variables. When the structure fails, the probability of failure can be obtained:

$$
\boldsymbol{P}_{f}=\frac{N_{f}}{N}
$$

Where $N_{f}$ represents the failure times $(g<0), N$ represent the simulation times.

Use the ratio of the expectation and standard deviation of the performance function to solve the reliability index:

$$
\beta=\frac{\mu_{g}}{\sigma_{g}}
$$

Where ${ }^{\beta}$ is the reliability index, ${ }^{\mu_{g}}$ is the expectation of the performance function, ${ }^{\sigma_{s}}$ is the standard deviation of the performance function.

\section{CONCENTRATED IMPACT EXPERIMENT}

\section{A. Experimental Facility}

Concentrated impact experiment was carried aimed at studying the dynamic reliability problems of the orthotropic rectangular membrane structures under concentrated stochastic impact load. Membrane purchased from a certain supplier of architectural membranes, and the material details were provided by the manufacturer, which is as shown in the Tab.1. Membranes need to be cut, heat bonded and punched.

TABLE I. SPECIFIC PARAmeters OF MEMBrane Material

\begin{tabular}{ccccc}
\hline Parameters & Size & Thicken & Density & $\begin{array}{c}\text { Elastic modulus } \\
\text { (warp/weft) }\end{array}$ \\
\hline Value & $\begin{array}{l}1200 \mathrm{~mm} \\
\times 800 \mathrm{~mm}\end{array}$ & $0.72 \mathrm{~mm}$ & $0.95 \mathrm{~kg} / \mathrm{m}^{2}$ & $1590 / 1360 \mathrm{MPa}$ \\
\hline
\end{tabular}

The incidence velocity and reflection velocity of ball are obtained by the two-way tachometer, and their difference value is impact velocity. The statistics shows that the impact velocity approximates Gaussian distribution and could be Gaussian fitted, which is consistent with the meteorological law of hail in the natural environment. The dynamic response of the membrane was measured by a non-contact laser displacement sensor, which obtained the displacement, velocity and acceleration. Measurement points were $\mathrm{C} 1$ point, $\mathrm{C} 2$ point and $\mathrm{C} 3$ point, the distribution of location as shown in Fig.2.
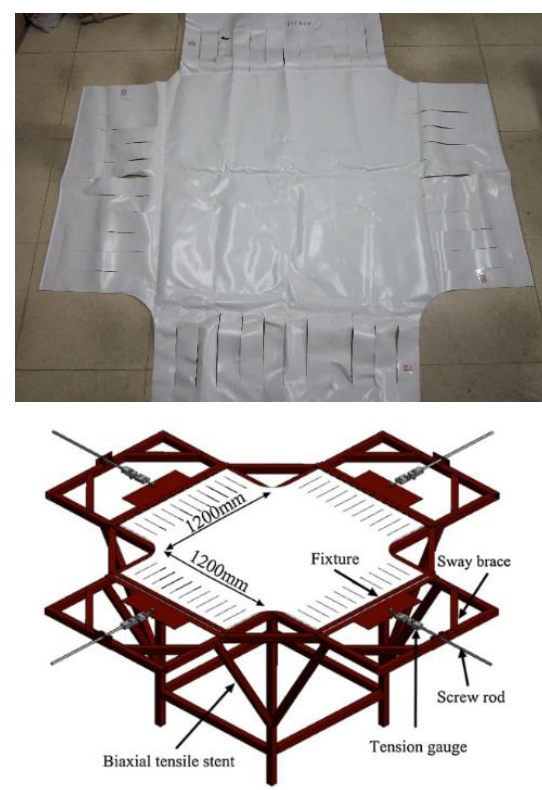

Figure 1. The membrane material and experimental facilities

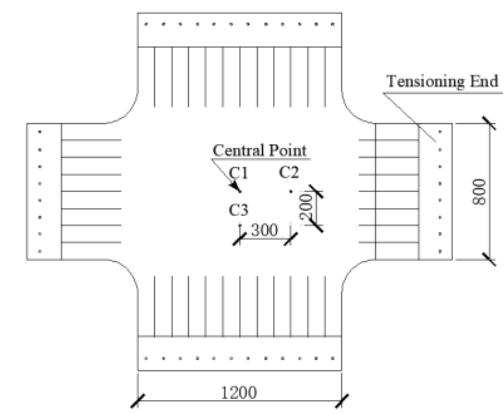

Figure 2. The membrane specimen and the measure point

\section{B. Analysis of the Impact Velocity}

The velocity difference value between the incidence velocity and the reflection velocity is studied. Experiments were performed for 160 times, which obtained sample data of 160 impact velocities . Ignore the failed tests, 146 sample data remain valid. These sample data were analyzed.

The least-squares method was used to process the impact velocity to obtain the probability density curve. According to the characteristic of sample distribution, it can be fitted to a Gaussian distribution. Assume its probability density function is:

$$
p_{\delta v}(\delta v)=a \exp \left[-\frac{(\delta v-E[\delta v])^{2}}{2 \sigma_{\delta v}^{2}}\right]
$$


Where $E[\delta v]$ is the expectation of the stochastic variable $\delta v, \sigma_{\delta v}$ is the standard deviation, $a$ is an constant.

Log terms on both sides:

$$
\ln \left(p_{\delta v}(\delta v)\right)=\ln a-\frac{1}{2 \sigma_{\delta v}^{2}}\left(\delta^{2} v-2 E[\delta v] \delta v+E^{2}[\delta v]\right)
$$

Let

$$
Y=\ln \left(p_{\delta v}(\delta v)\right), \quad a_{0}=-\frac{1}{2 \sigma_{\delta v}^{2}}, a_{1}=\frac{E[\delta v]}{\sigma_{\delta v}^{2}}, a_{2}=\ln a-\frac{E^{2}[\delta v]}{2 \sigma_{\delta v}^{2}}, y_{i} \text { is the }
$$

value of $Y$.The equation becomes:

$$
Y=a_{0} \delta^{2} v+a_{1} \delta v+a_{2}
$$

Now use polynomial fitting to determine $a_{0}, a_{1}, a_{2}$ so as to obtain the value of $E[\delta v], \sigma_{\delta v}, a$. The regular equations of polynomial fitting is:

$$
\left(\begin{array}{ccc}
n+1 & \sum_{0}^{n} \delta v_{i} & \sum_{0}^{n} \delta^{2} v_{i} \\
\sum_{0}^{n} \delta v_{i} & \sum_{0}^{n} \delta^{2} v_{i} & \sum_{0}^{n} \delta^{3} v_{i} \\
\sum_{0}^{n} \delta^{2} v_{i} & \sum_{0}^{n} \delta^{3} v_{i} & \sum_{0}^{n} \delta^{4} v_{i}
\end{array}\right)\left(\begin{array}{c}
\sum_{0}^{n} y_{i} \\
a_{1} \\
a_{0}
\end{array}\right)=\left(\begin{array}{c}
\sum_{0}^{n} \delta v_{i} y_{i} \\
\sum_{0}^{n} \delta^{2} v_{i} y_{i}
\end{array}\right)
$$

According to coefficient matrix of regular equations, the following algebraic equations are obtained:

$$
\begin{gathered}
\left(\begin{array}{ccc}
16 & 612.375 & 25034.51 \\
612.375 & 25034.51 & 1024834.44 \\
25034.51 & 1024834.44 & 42010507.80
\end{array}\right)\left(\begin{array}{l}
a_{2} \\
a_{1} \\
a_{0}
\end{array}\right)=\left(\begin{array}{c}
-43.4771 \\
-1777.32 \\
-72773.43
\end{array}\right) \\
a_{2}=-584.98, a_{1}=28.65, a_{0}=-0.35 \\
E[\delta v]=40.7,2 \sigma^{2}=2.843
\end{gathered}
$$

The probability density function is:

$$
p_{\delta v}(\delta v)=0.3346 \exp \left[-\frac{(\delta v-40.7)^{2}}{2.843}\right]
$$

Test the stability of the impact velocity in this experiment by rounds method. Among the sample data, remove the first and last data. This would not affect the result of the test. The remaining 144 data were divided into 9 groups with 16 data in each group. The values are shown in the Tab.2.

\section{Maximum of Deflection Response}

The advanced first-order second-moment method (AFOSM) and the Monte-Carlo method(MCS) are used to process the experimental model and the load model. The simulation times of MCS is 1000 . The failure probability and reliability index under different deflection thresholds are obtained as shown in Tab.3. This table shows that the probability of failure calculated by AFOSM is very close to that of MCS. Meanwhile, the Monte-Carlo method itself is a simulation method by generating large numbers of random numbers for calculation. The result is undoubtedly very accurate, which proves that AFOSM can be well applied to the calculation of the dynamic reliability of rectangular orthotropic membrane.

TABLE II. THE SIGN TABLE IN ROUNDS METHOD

\begin{tabular}{lccccc}
\hline $\begin{array}{c}\text { Mean in } \\
\text { group }\end{array}$ & 41.61 & 40.07 & 40.67 & 40.38 & 41.05 \\
\hline Sign & + & - & - & - & + \\
\hline $\begin{array}{c}\text { Mean in } \\
\text { group }\end{array}$ & 40.83 & 40.91 & 40.41 & 40.55 & \\
\hline Sign & + & + & - & - \\
\hline
\end{tabular}

\section{PARAMETER ANALYSIS}

This chapter mainly uses the theory and the numerical simulation method to study the reliability index and failure probability $\beta$ of the membrane calculated via first-second second-moment method and the Monte-Carlo method, whose simulation times are 1000 and 200 with the deflection threshold is $0.250 \mathrm{~mm}$ while the pretension force and mass of ball varying. And the influences of these two parameters on the structural failure and the differences among different methods are obtained.

\section{A. Effect of Pretension Force}

Fig. 3 and Fig. 4 show the change regulation of reliability under different pretension forces. As can be seen from the figures, the results obtained by AFOSM are in good agreement with those obtained by the Monte-Carlo method with 1000 simulation times. The results of the Monte-Carlo method with 200 simulation times show a certain degree of volatility caused by the lack of simulation times. The reliability index increased almost linearly with the increase of pre-tension in the early stage, but declined again at $7040 \mathrm{kN}$. Although increasing the pre-tension could reduce the amplitude of the vibration, the natural frequency is also changed, which leads to the case that pretension incensement is not large, the latitudinal-longitudinal coupling effect on the structure is dominant in reliability control. Therefore, when the maximum deflection of the structure approaches the threshold, it is necessary to increase the pretension to get it out of the dangerous range near the threshold. 


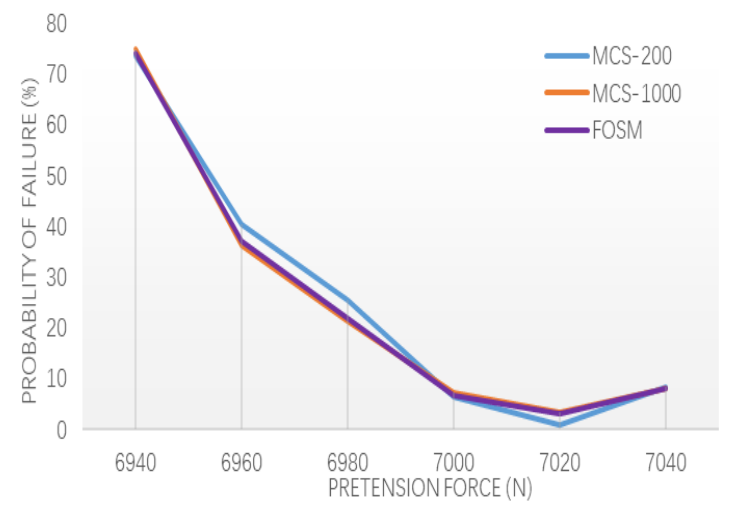

Figure 3. Relationship between probability of failure and pretension force.

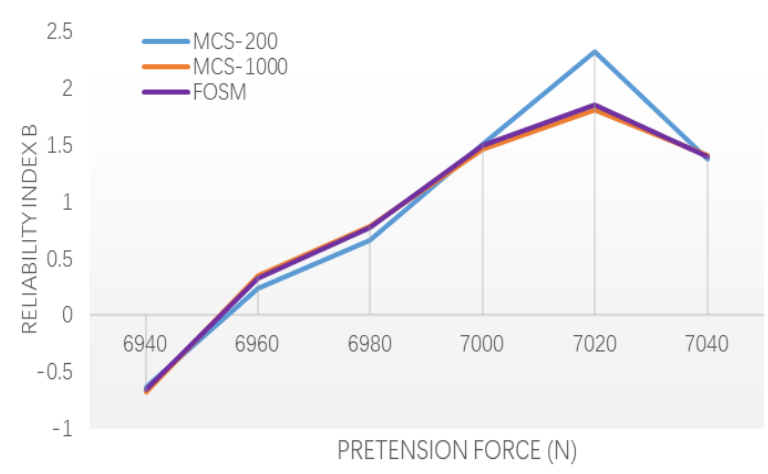

Figure 4. Relationship between reliability index $\beta$ and pretension force.

TABLE III. The ProbabILITY of FAILURE AND RELIABILITY INDEX B

\begin{tabular}{ccccc}
\hline $\begin{array}{c}\text { Threshold } \\
(\mathrm{mm})\end{array}$ & \multicolumn{2}{c}{ Probability of Failure $(\%)$} & \multicolumn{2}{c}{ Reliability Index $\beta$} \\
\hline 0.230 & FOSM & MCS & FOSM & MCS \\
0.235 & 91.19 & 92.30 & -1.3525 & -1.4255 \\
0.240 & 73.85 & 74.60 & -0.6386 & -0.6620 \\
0.245 & 47.00 & 48.10 & 0.0754 & 0.0476 \\
0.250 & 21.49 & 22.30 & 0.7895 & 0.7621 \\
0.255 & 6.63 & 6.30 & 1.5036 & 1.5301 \\
0.260 & 1.33 & 1.10 & 2.2178 & 2.2904 \\
0.265 & 0.17 & 0.30 & 2.9321 & 2.7478 \\
\hline
\end{tabular}

\section{B. Effect of Impact Mass}

Fig.5 and Fig.6 show the change regulation of reliability under different mass of impact body. It can be seen from the figure that the reliability index decreases linearly with the increase of the ball mass, and when the ball mass is less than $0.24 \mathrm{~g}$, the number of failure times simulated by the MCS method is 0 , that is, the reliability index is infinity, the structure is in an absolute safe state. Although the result calculated by the AFOSM can be expressed as a numerical value but not zero or infinity, the probability of failure in this case is a quite small value, so that the structure can be regarded as being safe. Therefore, when calculating the load for membrane structure design, increasing the design value of load by multiplying coefficients etc. is very advantageous for structural safety.

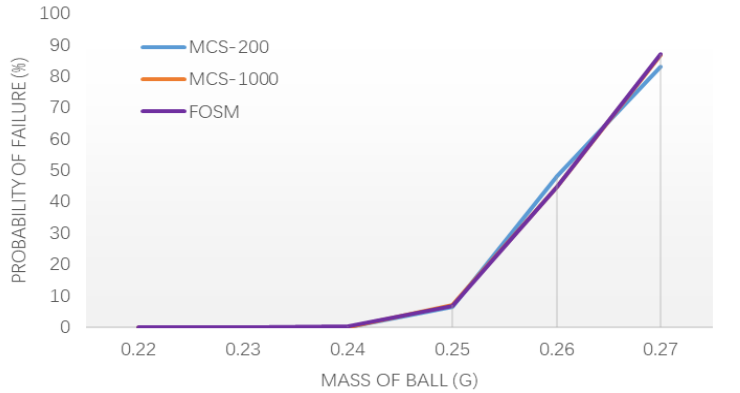

Figure 5. Relationship between probability of failure and pretension force.

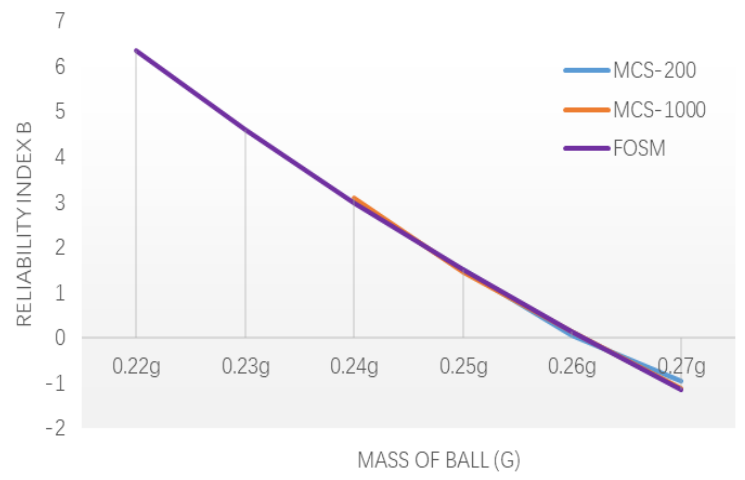

Figure 6. Relationship between reliability index $\beta$ and pretension force. 


\section{CONCLUSION}

In this paper, based on the first transgression criterion, the failure probability and dynamic reliability index of membrane structures are solved by applying the advanced first-order second-moment method. In addition, the dynamic reliability of stochastic vibration is studied experimentally, and the theoretical model is validated by theoretical and experimental comparison. The influence of parameters such as pretension and impact body mass on dynamic reliability is also discussed. The main conclusions are as follows:

(1) The advanced first-order second-moment method (AFOSM) is able to calculate the dynamic reliability of the membrane structure accurately.

(2) The membrane reliability generally increases linearly with the incensement of pretension force. However, due to the change of natural frequency, the pretension force increases slightly, which may reduce the reliability of the structure. When the maximum deflection of the structure approaches the threshold, it is necessary to increase the pretension to get it out of the dangerous range near the threshold.

(3) The membrane reliability decreases linearly with the incensement of impact body mass. When designing the load, increasing the design value of load by multiplying the coefficient can effectively improve the structural reliability.

(4) This theoretical and experimental study expands membrane vibration studies and provides references for the theoretical study of the reliability of membrane structures.

\section{ACKNOWLEDGMENT}

This research was supported by the grants from: Natural Science Foundation of China (No.51178485, No.51608060)
Chongqing Municipal Construction Committee, Construction and Scientific 2014 Project No. 10-5.

\section{REFERENCES}

[1] F. Khan, F. Sassani, B. Stoeber, Nonlinear behaviour of membrane type electromagnetic energy harvester under harmonic and random vibrations. Microsystem Technology, (2014) 20 (7) 1323-1335.

[2] Jie Li, Jian-bing Chen, Wen-liang Fan, The equivalent extreme-value event and evaluation of the structural system reliability. Structural Safety 29 (2007) 112-131.

[3] J.W. Fox, N.C. Goulbourne. Electriceld-induced surface transformations and experimental dynamic characteristics of dielectric elastomer membranes. Journal of the Mechanics and Physics of Solids, 57 (2009) 1417-1435.

[4] J.W. Fox, N.C. Goulbourne. Electriceld-induced surface transformations and experimental dynamic characteristics of dielectric elastomer membranes. Journal of the Mechanics and Physics of Solids, 57 (2009) 1417-1435.

[5] C. J. Liu, Z. L. Zheng, X. T. He. L-P perturbation solution of nonlinear vibration of prestressed orthotropic membrane in large amplitude. Mathematical Problems in Engineering, 2010 242-256.

[6] P.D. Gosling, B.N. Bridgens, L. Zhang, Adoption of a reliability approach for membrane structure analysis. Structural Safety, 40 (2013) $39-50$.

[7] Weiju Song, Yingmin Li, Nonlinear Vibration Analysis of Stochastic Thin Plates Based on Lindstedt-Poincaré Perturbation Method. Journal of Information \& Computational Science, 2015 12:6 24392450

[8] Z.L. Zheng, F.M. Lu, X.T. He, J.Y. Sun, C.X. Xie, C. He, Large displacement analysis of rectangular orthotropic membranes under stochastic impact load. International Journal of Structure Stability and Dynamics, 201616 (1) 928-936.

[9] CECS 158: 2015, Technical specification for membrane structures. Beijing, China Planning Press, 2016. 